\title{
A Unified Theoretical Description of the Thermodynamical Properties of Spin Crossover with Magnetic Interactions
}

\author{
Kamel Boukheddaden ${ }^{1}$, Masamichi Nishino ${ }^{2, *}$, Seiji Miyashita ${ }^{3}$, and François \\ Varret $^{1}$ \\ ${ }^{1}$ Laboratoire de Magnétisme et d'Optique, CNRS-Université \\ de Versailles/St. Quentin en Yvelines 45 Avenue des Etats Unis, F78035 \\ Versailles Cedex, France \\ ${ }^{2}$ Computational Materials Science Center, National Institute for \\ Materials Science, \\ Tsukuba, Ibaraki 305-0047, Japan \\ ${ }^{3}$ Department of Physics, Graduate School of Science, \\ The University of Tokyo, Bunkyo-Ku, Tokyo, Japan.
}

(October 9, 2018)

\begin{abstract}
After the discovery of the phenomena of light-induced excited spin state trapping (LIESST), the functional properties of metal complexes have been studied intensively. Among them, cooperative phenomena involving low spin-high spin (spin-crossover) transition and magnetic ordering have attracted interests, and it has become necessary to formulate a unified description of both phenomena. In this work, we propose a model in which they can be treated simultaneously by extending the Wajnflasz-Pick model including a magnetic interaction. We found that this new model is equivalent to Blume-Emery-Griffiths (BEG) Hamiltonian with degenerate levels. This model provides a unified description of the thermodynamic properties associated with various types of systems, such as spin-crossover (SC) solids and Prussian
\end{abstract}


blue analogues (PBA). Here, the high spin fraction and the magnetization are the order parameters describing the cooperative phenomena of the model. We present several typical temperature dependences of the order parameters and we determine the phase diagram of the system using the mean-field theory and Monte Carlo simulations. We found that the magnetic interaction drives the SC transition leading to re-entrant magnetic and first-order SC transitions.

Typeset using REVTEX 


\section{INTRODUCTION}

Recently the functionality of metal complexes attracts much interest. In particular, the cooperative phenomena where both spin-crossover (SC) and magnetic ordering are involved have been studied intensively. Molecular switching of inorganic solids is a typical issue of the vibronic lability of molecular units, originally introduced in chemistry by J.A. Ammeter $[1,2]$.

The concepts of molecular bistability were given by O. Kahn [2], and recent reviews on this subject can be found in the references [3-6]. In addition, due to the characteristic of reversible control of the magnetic properties by light, temperature, pressure, and magnetic field, SC and PBA compounds are considered to be promising candidates for information storage. [7-9].

system low-temperature sate High temperature State ref.

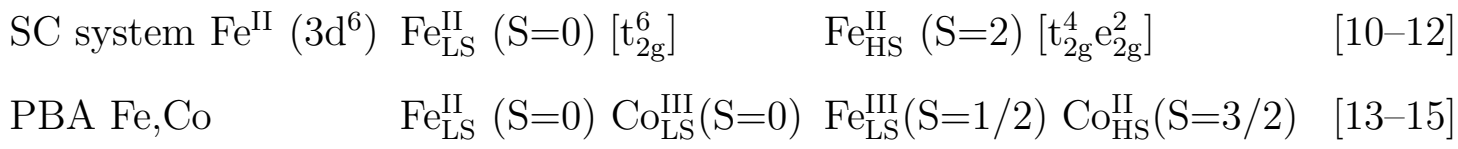

In SC, the electronic structure changes between the high spin state (HS) and and the low spin state (LS) in an atom which causes changes of magnetic property and also volume of the molecule. On the other hand, in PBA the charge transfer between Fe and Co causes a LS-HS (spin-crossover) transition of Co, and the magnetic moments of the Co and Fe couple antiferromagnetically in the high spin state (HS), which results in local ferrimagnetic moment. Although the LS-HS transition of PBA is different from simple SC transitions, we can regard PBA as a SC system in the broad sense. A new aspect of PBA is the magnetic ordering at low temperatures, where the magnetic interaction between spins is important.

As another type of phase transition due to the charge transfer, other new inorganic systems, showing magnetic changes after the spin transition, have been reported in literatures, such as mixed-valence iron complex $\left(\mathrm{nC}_{3} \mathrm{H}_{7}\right)_{4} \mathrm{~N}\left[\mathrm{Fe}^{\mathrm{II}} \mathrm{Fe}^{\mathrm{III}}(\mathrm{dto})_{3}\right]\left(\mathrm{dto}=\mathrm{C}_{2} \mathrm{O}_{2} \mathrm{~S}_{2}\right)$ [16,17], where the charge transfer causes a change of degeneracy, and a phase transition occurs between a high temperature 
and low temperature states. In this system, a magnetic transition is also observed at a low temperature. There, the spin-crossover of each atom does not occur. However, the entropy effect plays an important role in the structure change associated with the electronic configuration. It is an interesting problem to study the mechanism of combined phenomena of the structure change and magnetic transition.

In SC transition, a cooperative distortion of the lattice occurs, which is associated with a large change in the average metal-ligand distance. Indeed it increases about $10 \%$ at the molecular level in the conversion from the LS to HS state which causes a volume expansion of the molecules. Thus, the SC phenomenon at the microscopic scale is due to the coupling between the electronic and the vibrational structures [18] of the molecules. That is the intra-molecular vibronic coupling. The coupling between the lattice distortion and the change of the electronic states of the molecules induces the cooperativity of SC solids. This is the microscopic origin of the elastic interaction which is responsible for the SC transition. From the experimental point of view, optical and magnetic properties change during the SC transition, so that the switching properties can be followed by magnetic or optical (absorption, reflectivity) techniques.

For the analysis of these structural transition, it is largely admitted that the bistable properties at the molecular level are adequately described through a molecular configurational diagram, i.e. a plot of adiabatic energies versus distortion coordinate of the molecular system. In Fig.1, we show a unified three-states configurational diagram suited to $\mathrm{SC}$ and $\mathrm{PBA}$ in the case of the low spin (LS) ground state. It is noteworthy that the effect of environment in molecular solids affects the configurational diagram. For example, an external pressure mainly increases the $E(\mathrm{HS})-E(\mathrm{LS})$ energy gap, and thus reduces energy barrier between HS and LS states in SC solids. Consequently, it raises the transition temperature $\left(T_{\text {eq }}\right)$ and it decreases the lifetime of the metastable HS state [19-21]. In the case of PBA, the same effect of pressure is also expected on the spin transition of cobalt [22], which would cause variation of the magnetic properties under high pressures. In the 
mixed-valence iron complex $\left(\mathrm{nC}_{3} \mathrm{H}_{7}\right)_{4} \mathrm{~N}\left[\mathrm{Fe}^{\mathrm{II}} \mathrm{Fe}^{\mathrm{III}}(\text { dto })_{3}\right]\left(\mathrm{dto}=\mathrm{C}_{2} \mathrm{O}_{2} \mathrm{~S}_{2}\right)$, the pressure effect has been reported [23]. 


\section{FIGURES}

FIG. 1. A unified three-states configurational diagram. The non-degenerate ground state is of low spin and the excited degenerate states are of high spin.

To describe SC systems, a two-states Ising-like model proposed by Wajnflasz and Pick (WP) [24] is currently used. In this phenomenological model, the HS and LS microscopic states of the molecule are associated, respectively, with the eigenvalues +1 and -1 of the fictitious spin operator $s$ with their respective degeneracies $g_{+}$and $g_{-}$. Here, we denote by $g=\frac{g_{+}}{g_{-}}>1$ the degeneracy ratio, by $\Delta>0$ the ligand field energy (such that the LS state is the ground state at $0 \mathrm{~K}$ ), and by $J^{\prime}$ the interaction between the molecules. $J^{\prime}$ is assumed to have an independent value on the spin states. Taking into account the isomorphism [25] between the Ising model with degenerate levels and an Ising model under a temperature dependent "field" [26], the WP Hamiltonian is written as

$$
H=-J^{\prime} \sum_{i \neq j} s_{i} s_{j}+\left(\Delta-\frac{k_{\mathrm{B}} T}{2} \ln g\right) \sum_{i} s_{i},
$$

where $\sum_{i \neq j}$ is the sum over the interacting neighbors and $s$ takes 1 (HS) or -1 (LS). $J^{\prime}>0$ is the interaction parameter between the SC units.

As we mentioned before, the interaction in SC solids originates from elastic strength [27]. However, in the WP model it is expressed through the phenomenological term $-J^{\prime} \sum_{i \neq j} s_{i} s_{j}$. Here $J^{\prime}$ is not an exchange magnetic coupling. This term expresses the situation that an attractive force (i.e. a ferroelastic interaction) acts between neighboring sites in the same state (LS-LS or HS-HS) and a repulsive force acts between those in different states (LS-HS), which leads to bistability of the LS and HS states. A more realistic treatment which includes a spin-phonon interaction leads to obtain a similar Hamiltonian as (1) with effective parameters $J^{\prime}$ and $\Delta$ which depend on the distortions of the molecules at sites $i$ and $j$ [28]. Thus, it is important to consider here the parameters $J^{\prime}$ and $\Delta$ as effective parameters which have elastic origin, even if they are taken as constants in the model in order to make the present treatment easy. 
The degeneracy ratio $(g)$ between the HS and LS states is related to the molar entropy change $(\Delta S)$ due to the total spin-conversion which is given by $\Delta S=$ $N k_{\mathrm{B}} \ln g$, where $k_{\mathrm{B}}$ is the Boltzmann constant and $N$ the Avogadro's number. Because of both electronic, vibrational (intramolecular) and phonon factors, the experimental entropy changes by $\Delta S=30-70 \mathrm{JK}^{-1} \mathrm{~mol}^{-1}$ [4]. This corresponds to the following degeneracy values $g=36-4500$, which indicates that $g \gg 1$. Generally, $g$ depends on temperature through the temperature dependence of the phonon density of the lattice [29]. Here, we simply take $g$ as a constant.

The ratio of molecules in the high spin state, called HS fraction, is denoted by $n_{\mathrm{HS}}$, which is expressed as a function of the "fictitious magnetization" $<s>$ such as $n_{\mathrm{HS}}=(<s>+1) / 2$. Despite of the drastic simplification of the realistic vibronic levels scheme by the two-level system, this model permits to describe with amazing success most of the quasi-static properties of the SC solids. For example, it explained consistently the change from a smooth transition to a first order transition $[24,26,30]$ of spin conversion between the LS and HS states as a function of the parameters of the model. It also well explained the two-step spin crossover behavior [31-33].

In order to study the magnetic properties in PBA solids, etc., we need to go beyond the 2-states model. In previous studies [34,35], focusing on the condition of the reversible switching between nonmagnetic LS state and the magnetic metastable ordered HS state which was observed in PBA [13], the following three state model called Blume-Capel model [36,37] was investigated:

$$
H=-J \sum_{i \neq j} S_{i} S_{j}+\Delta \sum_{i} S_{i}^{2}
$$

In this model, the spin operator $S_{i}$ has three states: $-1,0,+1$, where $0( \pm 1)$ is associated with the LS nonmagnetic (HS magnetic) state. Here, $J$ is really an exchange magnetic coupling and $\Delta$ plays a role of a ligand field. Reversible switching was demonstrated by a suitable change of parameters of the model assuming photon's effect is renormalized into the parameters. In addition, from a dynamical viewpoint, it has been pointed out that existence of multi-time scale dynamics is essential for 
the switching in both directions (magnetic $\leftrightarrow$ diamagnetic). That is, the difference between the time scales of the spin flip process and of structural change (LS $\leftrightarrow \mathrm{HS}$ ) process is important. This concept is very important to investigate photomagnetism of PBA [38].

To reproduce the temperature-induced SC properties of PBA, the information of the difference in degeneracies between the high temperature and the low temperature phases and the nonmagnetic interaction between molecules (which is originated from the lattice) have to be taken into account. This is the motivation of this paper. Here, we propose a model taking into account both mechanisms of the temperatureinduced SC transition and of the magnetic transition. Then, we extend the 3states model [34,35] (given by the Hamiltonian (2)) to include the mechanism in the Wajnflasz and Pick model (Hamiltonian (1)). As we will see in the next section, this present model can be regarded as an extended Blume-Emery-Griffiths (BEG) Hamiltonian [39].

It is worth noting that for the elastic interaction between molecules, alternative scenarios to describe the SC phenomenon have been proposed recently. Some of them are based on pseudo-spins coupled by springs [28]. The originality of this approach is that the elastic constant between two neighboring atoms depends on their electronic states. Indeed, due to the expansion of the lattice in the HS state, the elastic force constants are weaker in the HS state than in the LS state. This approach leads to a Hamiltonian which is equivalent to an Ising-like model in which the interaction parameter depends on the local distortions of the molecules. An extension of this scenario including the intramolecular potential energy of the molecules has been published recently by one of the authors [40]. However, due to the complicated structure of the Hamiltonians, these models have been investigated only in the one dimensional case, but we expect new development of this kind of descriptions in near future.

This paper is organized as follows: in section II we introduce the model, section III is devoted to study the equilibrium properties obtained by a mean-field theory. In 
section IV, results of Monte Carlo simulations are presented. In section V, we discuss the obtained results in comparison with available experiments in the literature and we conclude.

\section{THE HAMILTONIAN}

Here we adopt a model based on a three states Ising-like Hamiltonian of $S=$ $0,+1,-1$. The state $S=0$ denotes the non-magnetic state, which corresponds to the electronic configuration $\mathrm{Fe}_{\mathrm{LS}}^{\mathrm{II}} \mathrm{Co}_{\mathrm{LS}}^{\mathrm{III}}$ in the case of PBA, and simply $\mathrm{Fe}_{\mathrm{LS}}^{\mathrm{II}}$ for $\mathrm{SC}$ systems. The states $S= \pm 1$ are associated with magnetic states. The magnetic state corresponds to the ferrimagnetic state $\mathrm{Fe}_{\mathrm{LS}}^{\mathrm{III}} \mathrm{Co}_{\mathrm{HS}}^{\mathrm{II}}$ for $\mathrm{PBA}$ compounds, and $\mathrm{Fe}_{\mathrm{HS}}^{\mathrm{II}}$ in the case of SC solids. Due to large expansion of the coordination sphere of the molecule during the spin transition, the vibrational structure [4] and the electronic properties between the HS and LS states are strongly affected. Consequently, the degeneracies of the non-magnetic state and the magnetic state are different. Let us denote by $u$ and $r$ the degeneracies of the states $S=0$ and $S= \pm 1$, respectively, and by $g$ the ratio $\frac{r}{u}$. Here we assume that the degeneracies of the states with $S= \pm 1$ are the same and $g$ is temperature independent.

Because the high and low spin states are described by $S= \pm 1$ and $S=0$, respectively. The Wajnflasz and Pick model (1) in the present three states approach, in which the degeneracies are included as entropic terms [24,26,25,41], is given by

$$
H_{\mathrm{WP}}=-J^{\prime} \sum_{i \neq j}\left(2 S_{i}^{2}-1\right)\left(2 S_{j}^{2}-1\right)+\left(\Delta-\frac{k_{\mathrm{B}} T}{2} \ln g\right) \sum_{i}\left(2 S_{i}^{2}-1\right) .
$$

Adding the magnetic interaction $-J \sum_{i \neq j} S_{i} S_{j}$ and the Zeemann energy $-h \sum_{i} S_{i}$, we obtain the general Hamiltonian:

$$
H=-J \sum_{i \neq j} S_{i} S_{j}-h \sum_{i} S_{i}-K \sum_{i \neq j} S_{i}^{2} S_{j}^{2}+\left(D-k_{\mathrm{B}} T \ln g\right) \sum_{i} S_{i}^{2}+\text { const. }
$$

where $K=4 J^{\prime}(>0)$ and $D=2 \Delta+4 z J^{\prime}>0(z:$ the coordination number). Here only the nearest neighbor interactions are considered for the pairing $i \neq j$. The magnetic interaction between the spins is only relevant when both spins $S_{i}$ and $S_{j}$ 
are in the magnetic state, i.e. $S= \pm 1$. If we do not consider the degeneracies, i.e., $u=r=1$ and $g=1$, this Hamiltonian corresponds to the well known BEG model. [39] Here, we adopt the form of Eq. (4) to refer to past works on BEG model. Indeed, BEG Hamiltonian was extensively studied to describe the thermodynamical properties of the tri-critical point in $\mathrm{He}^{3}-\mathrm{He}^{4}$ mixtures [39]. Later, Sivardière and Lazerowitch [42] used an extended version to lattice gas model of BEG Hamiltonian to study the condensation and phase separation in binary fluids. It is also interesting to mention that even recently, BEG Hamiltonian was also applied [43] to describe the ferroelectric order thermally induced in donor-acceptor materials like TTF-TCNQ [44]. This kind of materials are also intensively studied for their photo-induced properties [45].

\section{EQUILIBRIUM PROPERTIES}

In experiments, so far the spin crossover and magnetic ordering in PBA take place in the range of temperature $10-300 \mathrm{~K}$. As typical values of the parameter of the model, extracted from the experiments $[15,46]$, we can refer to the exchange interaction $z J \simeq 20 \mathrm{~K}$, the ligand-field energy gap $D \approx 500 \mathrm{~K}$, the elastic interaction $z K$ is about $100-200 K$ and the degeneracy ratio $g \simeq 100-200$. Here $z$ is the number of the nearest neighbor pairs. The present model describes the systems of SC and PBA solids as special cases:

(i) for SC systems, the magnetic interaction between the magnetic HS units is negligible because of the large distance between them $(\approx 10 \AA)$ [47].

(ii) In the case of PBA, all the terms of the model should be taken into account, because they are effectively present. However, as far as we concern the equilibrium thermodynamics, only the electron-transfer contributes to the thermal bistability between the states $\mathrm{Fe}_{\mathrm{LS}}^{\mathrm{II}} \mathrm{Co}_{\mathrm{LS}}^{\mathrm{III}}$ and $\mathrm{Fe}_{\mathrm{LS}}^{\mathrm{III}} \mathrm{Co}_{\mathrm{HS}}^{\mathrm{II}}$. Indeed around the spin crossover transition temperature, experimentally around $200-300 \mathrm{~K}$, there is no magnetic ordering. This fact suggests that the magnetic coupling $z J$, which is about $20 \mathrm{~K}$, is very small 
in comparison to the ligand-field energy gap $D \approx 500 \mathrm{~K}$. However, in the near future, because of an amazing developments of synthesis of materials in this subject, we may expect new systems in which the magnetic and SC properties could compete. Thus, we study a general structure of the phase diagram caused by $K$ and $J$ in the present paper.

\section{A. The SC case}

As we mentioned above, in the case of SC, the magnetic interaction is irrelevant and the Hamiltonian (4) without the magnetic field and magnetic interaction can be written in the following form

$$
H_{e q}=-K \sum_{i \neq j} S_{i}^{2} S_{j}^{2}+\left(D-k_{\mathrm{B}} T \ln g\right) \sum_{i} S_{i}^{2}
$$

It is straightforward to see that a flipping of any spin $\left(S_{i} \rightarrow-S_{i}\right)$ does not change

the Hamiltonian. Therefore, as expected, the magnetization $m=<S>$ should be zero at any temperature. Introducing a new variable $\sigma_{i}=2 S_{i}^{2}-1$, the Hamiltonian 5 reduces to WP model [24], which leads to a first order phase transition as it is well known from the literature $[26,30,48,49]$ :

$$
H_{\sigma}=-\frac{K}{4} \sum_{i \neq j} \sigma_{i} \sigma_{j}+\frac{D-k_{\mathrm{B}} T \ln 2 g-z K / 2}{2} \sum_{i} \sigma_{i}+\text { const. }
$$

In a mean-field approximation, the order parameter of the system is $q=<S^{2}>$. It corresponds exactly to the HS fraction of molecules in the system. The selfconsistent equation accounting for the thermally driven first-order transition between the macroscopic LS $(q \simeq 0)$ and HS $(q \simeq 1)$ states, is given by

$$
k_{\mathrm{B}} T=\frac{D-z K q}{\ln \left(\frac{2 g(1-q)}{q}\right)} .
$$

So-called transition temperature $T_{\text {eq }}$ is obtained by setting $q=1 / 2$ in the equation (7), or more simply by setting the effective field $D-k_{\mathrm{B}} T \ln 2 g-z K / 2$ of the Hamiltonian (6) equal to zero. Thus we have 


$$
k_{\mathrm{B}} T_{\mathrm{eq}}=\frac{D-z K / 2}{\ln 2 g}
$$

It is interesting to notice that occurrence of the first-order transition is only possible when the condition $T_{\text {eq }}<T_{\mathrm{I}}$, where $T_{\mathrm{I}}$ denotes the critical temperature of the pure spin $1 / 2$ Ising model in the same lattice. In the mean-field approximation, the Curie temperature is given by $T_{\mathrm{I}}=z K / 4 k_{\mathrm{B}}$.

Two types of behavior of the order parameter can be obtained in the mean-field approach depending on the ligand-field energy gap $D$ and the interaction $K$ :

(i) a first-order spin-crossover transition, with a hysteresis loop, when $z K>$ $4 D /(2+\ln 2 g)$

(ii) a smooth conversion between the two states when $z K<4 D /(2+\ln 2 g)$.

The temperature dependence of $q$ is summarized in Fig.2.

FIG. 2. Temperature dependence of the high spin fraction $q$ for values of the energy gap $D$. From the left to the right, $D=90 \mathrm{~K}, 100 \mathrm{~K}, 120 \mathrm{~K}$ and $150 \mathrm{~K}$ with the fixed values of parameters $K=90$, and $g=10$. Smooth (first-order) transitions are obtained for $D>100 \mathrm{~K}(D<100 \mathrm{~K})$. The part of the curves with negative slope $d q / d t$ represents unstable states.

\section{B. General case.}

Here, we consider the general model of SC solids in which the magnetic moments of the HS state interact magnetically, which may cause a magnetic order. Therefore, in this section we consider the Hamiltonian (4) with various parameters. The model imposes two order parameters $m=<S>$ and $q=<S^{2}>$, respectively, associated with the magnetization and the high spin fraction in the system. If $K \gg J$ as the case of PBA, we can deal with a multi-scale energy problem in the present frame work: high energy scale for the spin-crossover phenomena (at high temperature), low energy scale for magnetic phenomena (at low temperature).

Now, let us study properties of the system (4) by a mean-filed theory (MFT) which gives the phase diagram of the model. The one site Hamiltonian for this 
system is given by

$$
H_{i}=-(z J m+h) S_{i}-\left(z K q-D+k_{\mathrm{B}} T \ln g\right) S_{i}^{2}
$$

The associated mean-field free energy per site is given by

$$
F(m, q, T)=\frac{1}{2} z J m^{2}+\frac{1}{2} z K q^{2}-k_{\mathrm{B}} T \ln \left(1+e^{\beta\left(z K q-D+k_{\mathrm{B}} T \ln 2 g\right)} \cosh \beta(h+z J m)\right) .
$$

Minimizing the free energy (10) with respect to the order parameters $m$ and $q$, we easily obtain the following coupled self-consistent equations on $m$ and $q$,

$$
m=\frac{\sinh \beta(h+z J m)}{e^{-\beta\left(z K q-D+k_{\mathrm{B}} T \ln 2 g\right)}+\cosh \beta(h+z J m)}=q \tanh \beta(h+z J m)
$$

and

$$
q=\frac{\cosh \beta(h+z J m)}{e^{-\beta\left(z K q-D+k_{\mathrm{B}} T \ln 2 g\right)}+\cosh \beta(h+z J m)} .
$$

If $J=h=0$, we reproduce naturally the case of SC previously studied. Equations $(9,11,12)$ have the same structure as that found in BEG model. However, due to the temperature dependent ligand-field (anisotropy in BEG model) we expect new behavior for the order parameters $m$ and $q$ and also a different phase diagram. Putting $g=1$ gives exactly the same self-consistent equations as that published by Mukamel and Blume [50] in their study of the BEG model to describe the thermodynamical properties of the tricritical point in $\mathrm{He}^{3}-\mathrm{He}^{4}$ mixtures. Our aim here is to analyze the effect of the magnetic interaction on the spin-crossover phenomena $(g \gg 1)$. Therefore, we study the following two cases:

1. $J=0, h \neq 0, K \neq 0, D \neq 0$;

2. $J \neq 0, h=0, K \neq 0, D \neq 0$;

$$
\text { 1. } J=0, h \neq 0, K \neq 0, D \neq 0 \text { case }
$$

This case corresponds to the situation of the SC system under a magnetic field $[51,52]$; the spins are too far to interact each other. In such situation, the system is 
paramagnetic or diamagnetic, and the magnetization has no strong effect. However, we study the effect of the magnetic field on the transition temperature and on the hysteresis loop in detail. In the present situation, the self-consistent equations $(11,12)$ become

$$
m=q \tanh \beta h
$$

and

$$
q=\frac{\cosh \beta h}{e^{-\beta\left(z K q-D+k_{\mathrm{B}} T \ln 2 g\right)}+\cosh \beta h} .
$$

It is clear from Eqns. (13 and 14) that the magnetization depends linearly on the HS fraction in the system. In the limiting case, where the magnetic field is very small in comparison with the transition temperature, the magnetization is $m \simeq q \beta h$. In the following, we will demonstrate that the magnetic field stabilizes HS (magnetic) state and decreases the transition temperature $T_{\text {eq }}$ of the system. So, we expand the equation (14) around the transition temperature of the first-order transition, for which $q=1 / 2$, by considering that $\beta h \ll 1$. After some simple calculations, we arrive at the following expression of the field dependence of transition temperature $T_{\text {eq }}(h)$

$$
T_{\text {eq }}(h)=T_{\text {eq }}(0)-\frac{h^{2}}{D-z K / 2} .
$$

Here, $T_{\text {eq }}(0)=\frac{D-z K / 2}{k_{\mathrm{B}} \ln 2 g}$ is the transition temperature of the system under zero applied magnetic field. The shift of the transition temperature

$$
\Delta T_{\mathrm{eq}}=-\frac{h^{2}}{k_{\mathrm{B}} T_{\mathrm{eq}}(0) \ln 2 g}=-\frac{h^{2}}{\Delta H(0)}
$$

depends on the ratio between the square of the magnetic field and the enthalpy change, $\Delta H(0)$, at the transition. These results are in good agreement with available experimental results and the microscopic analysis on $\mathrm{SC}[52,53]$ phenomena. It follows from this result that, lowering the transition temperature $T_{\text {eq }}(0)$ enhances the magnetic field effect on SC systems. 
FIG. 3. Effect of the 'magnetic' field $h(h=0 \mathrm{~K}, h=40 \mathrm{~K})$ for $K=200 \mathrm{~K}, D=300 \mathrm{~K}$, $g=150)$ on the temperature dependence of the HS fraction $q(T)$ in the case of the first order phase transition. The magnetic field stabilizes the HS phase and polarizes the magnetic system in which it induces a nonzero magnetization (dashed line).

On a hysteresis loop, see Fig.3, the magnetic field has as effect to shift the whole hysteresis to the low temperature side, by acting differently on the ascending and descending metastable branches. Denoting, by $T_{h}^{+}\left(T_{0}^{+}\right)$and $T_{h}^{-}\left(T_{0}^{-}\right)$, the transition temperatures under the field $h(h=0)$, at the ascending and descending branches, respectively, one can deduce approximately from the equation (15) that the associated shifts are given

$$
k_{\mathrm{B}} \Delta T^{+}=-\frac{h^{2}}{T_{0}^{+} \ln 2 g} \text { and } k_{\mathrm{B}} \Delta T^{-}=-\frac{h^{2}}{T_{0}^{-} \ln 2 g} .
$$

Since $T_{0}^{+}>T_{0}^{-}$, it follows that $\Delta T^{-}>\Delta T^{+}$, which indicates that the magnetic field causes a non-symmetric distortion of the hysteresis loop of SC solids by stabilizing the HS and destabilizing the LS state.

To give an order of magnitude of the shift of the hysteresis loop, we consider the case of

$$
\mathrm{Co}^{I I}\left(\mathrm{H}_{2}(\text { fsa })_{2} \mathrm{en}\right)(\mathrm{py})_{2} \quad\left[\mathrm{H}_{2}(\text { fsa })\right. \text { en }
$$

N,N'-ethylene bis(3-carboxysalicylaldimine,py) = pyridine] spin-crossover solid [54] which shows an abrupt thermally induced spin-crossover transition centered at $126 \mathrm{~K}$. For this system, the magnetic spin moments corresponding to the HS and the LS states are $S_{\mathrm{H}}=3 / 2$ and $S_{\mathrm{L}}=1 / 2$, and the energy gap is $700 \mathrm{~K}$ [55]. In our Hamiltonian, the parameter $h$ denotes $h=g_{\mathrm{L}} \mu B S$, where $\mu$ is the Bohr magneton, and $B$ the applied magnetic field. Here $g_{\mathrm{L}}$ is the Landé factor $g_{\mathrm{L}}=2$ for both LS and HS states. For $\mathrm{Co}^{I I}\left(\mathrm{H}_{2}(\mathrm{fsa})_{2} \mathrm{en}\right)(\mathrm{py})_{2}$, we found that the shift $\Delta T_{\text {eq }} \approx-4.9 \mathrm{~K}$ under a static magnetic field of 32 Tesla. 


$$
\text { 2. } J \neq 0, h=0, K \neq 0, D \neq 0 \text { case. }
$$

In this case, the HS units created thermally or optically interact through the exchange coupling $J$. We study effects of this magnetic interaction on the charge transfer involving SC transition, under zero applied magnetic field. In MFT, the thermodynamical properties are obtained by solving the following coupled self-consistent equations:

$$
m=q \tanh \beta z J m
$$

and

$$
q=\frac{\cosh \beta z J m}{e^{-\beta\left(z K q-D+k_{\mathrm{B}} T \ln 2 g\right)}+\cosh \beta z J m} .
$$

Here, it is clear from the equation (18) that the system may order magnetically, depending on the thermal behavior of the order parameter $q$. Indeed, at Curie temperature $T_{\mathrm{M}}$, we have

$$
q\left(T_{\mathrm{M}}\right) \beta_{M} z J=1
$$

It is interesting to notice that the thermal behavior of this complicated situation may be discussed through the comparison of the transition temperatures of the magnetic and spin-crossover systems. At Curie temperature $T_{\mathrm{M}}$, the magnetization $m(T)$ shows a second-order phase transition, and the HS fraction $q(T)$ exhibits a singularity.

Below $T_{\mathrm{M}}$ spontaneous magnetization appears where the system is globally in the HS phase. As the temperature decreases, the HS fraction decreases smoothly and the magnetization disappears at $T_{\mathrm{C}}\left(<T_{\mathrm{M}}\right)$ with a second-order transition for small value of $J$ where $q(T)$ exhibits a singularity. When $J$ is large, the magnetization remains until $T_{\text {eq }}$ and there disappears discontinuously at the first order phase transition temperature. 
FIG. 4. Temperature dependence of $m(T)$ (thin curves) and $q(T)$ (bold and dashed curves) in the case $z K=90 \mathrm{~K}, D=150 \mathrm{~K}$ and $g=10$ for the following values of the magnetic interaction. From the bottom to the top: $z J=48 \mathrm{~K}, z J=57.7 \mathrm{~K}, z J=58 \mathrm{~K}$, $z J=59 \mathrm{~K}, z J=61 \mathrm{~K}$, and $z J=63 \mathrm{~K}$. The solid bold curve denotes the HS fraction $q$ for $J=0 \mathrm{~K}$, in which $m=0$. Note that the magnetization $m(T)$ is equal to zero in the case $z J=48 K$.

When the SC and magnetic transition temperatures are of the same order of magnitude, the magnetic system (i.e., $m(T)$ ) perturbs the "lattice" properties (i.e., $q(T)$ ) as seen in Fig. 4, where the bold solid line denotes the temperature dependence of the order parameter $q$ for $J=0$. There all the simulations have been performed varying the magnetic coupling $J$ at fixed values of $K$ and $D: z K=90 \mathrm{~K}$ and $D=150 \mathrm{~K}$. For small values of $J$, as $z J=48 \mathrm{~K}$, no magnetization appears and only smooth SC transition occurs. When $z J$ has values between 57.7 and 59 for example, we observe a second-order re-entrant phase transition on the magnetization $m$ with the transition temperatures $T_{\mathrm{C}}$ and $T_{\mathrm{M}}$. The associated HS fraction shows singularity at each transition temperature. Increasing $z J$, such as $z J=63 \mathrm{~K}$, the magnetization presents a first-order transition at $T_{\mathrm{C}}$ and a second-order transition at $T_{\mathrm{M}}$. In such case, the HS fraction exhibits a first-order transition at $T_{\mathrm{C}}$ and a singularity at $T_{\mathrm{M}}$. Thus, it appears clearly that the magnetic coupling causes the first-order transition in the elastic properties (i.e., in $q(T)$ ). In the following we discuss the three regions identified in Fig. 4:

(1) a high temperature region in which $q \simeq 1, m=0$, corresponding to a paramagnetic HS state;

(2) an intermediate region $T_{\mathrm{C}}<T<T_{\mathrm{M}}$, where $q>1 / 2, m \neq 0$, indicating the existence of a predominant HS state with a ferromagnetic order;

(3) at low temperature $T<T_{\mathrm{C}}$, we have a phase of LS. There, we find two subregions. At relative high temperature region, $q$ is still large, where many high spins remain, which are made of isolated magnetic states $S= \pm 1$ in a sea of diamagnetic 
states $S=0$ where the system is paramagnetic. On the other hand, at low temperature $q$ is very small and the system is almost the perfect diamagnetic state. In this low temperature phase in which $m=0$ and $q<1 / 2$, we obtain a reentrant magnetic phase. Between the regions of (2) and (3), we find a first order SC phase transition driven by the magnetic interaction. Due to the magnetic interaction, the high spin fraction $q$ at the transition temperature $T_{C}$ is different from the usual value $q=1 / 2$ obtained for $J=0$.

In the present situation, i.e. case (3), both $m$ and $q$ undergo phase transitions. For the magnetization, when the temperature increases, magnetic property changes as the dia- $\rightarrow$ para- $\rightarrow$ ferro-magnetic and then paramagnetic state. If $J$ is large, the low temperature phase becomes ferromagnetic and then we have the change; the ferro- $\rightarrow$ to para-magnetic states. Concerning the high spin $q$, its temperature dependence can be either a first-order transition or a continuous conversion. These results obtained by MFT will be studied also by MC simulations in the next section.

To investigate the phase diagram of the SC system with magnetic interactions; we first expand the equations (18) and (19) around the magnetic transition temperature $T_{\mathrm{C}}$ (second-order case) or $T_{\mathrm{M}}$ for which $m \approx 0$. After some analysis, we arrive easily to the following equation, giving the transition line in the $(z J, T)$ plane

$$
d-\frac{t_{c}}{j}=t_{c} \ln \left(2 g \frac{j-t_{c}}{t_{c}}\right)
$$

with $d=\frac{D}{z K}, t_{c}=\frac{T_{\mathrm{C}}}{z K}$ and $j=\frac{J}{K}$. Here the reduced critical temperatures corresponding to $T_{\mathrm{C}} / z K$ and $T_{\mathrm{M}} / z K$ are denoted by the same quantity $t_{c}$.

In order to observe the re-entrant phase transitions with the present model, the function $j=f\left(t_{c}, d\right)$ must have a minimum. Due to the self-consistent structure of equation (21), it is difficult to predict analytically the coordinates $\left(t_{\text {crit }}, j_{\text {crit }}\right)$ of this minimum. Let us consider separately the left and right sides of the equation (21), and denote these two quantities by $f_{1}\left(t_{c}\right)=d-\frac{t_{c}}{j}$ and $f_{2}\left(t_{c}\right)=t_{c} \ln \left(2 g \frac{j-t_{c}}{t_{c}}\right)$. At the critical temperature, we must have $f_{1}\left(t_{c}\right)=f_{2}\left(t_{c}\right)$ and $f_{1}^{\prime}\left(t_{c}\right)=f_{2}^{\prime}\left(t_{c}\right)$, where the $f^{\prime}$ denotes the derivative of $f\left(t_{c}\right)$. These two relations lead to the following transition 
line for the critical points

$$
d+j_{\text {crit }}-1=j_{\text {crit }} \ln \left(2 g \frac{j_{\text {crit }}}{d}\right) .
$$

Solving numerically the last equation, using $d=D / z K=150 / 90=5 / 3$ and $g=10$, we obtain $j_{\text {crit }} \approx 0.64$, which gives $z J_{\text {crit }}=57.6 \mathrm{~K}$. This value is in very good agreement with the thermal behavior of the order parameters of Fig. 4. These results are summarized in the phase diagram of a SC system including magnetic interaction between the HS units, depicted in Fig. 5. A reentrant phase transition appears for $z J_{\text {crit }}=57.6 \mathrm{~K}<z J<z J_{0}=210 \mathrm{~K}$ where $j_{0}=2.33$. The magnetic phase appears from $T_{\mathrm{C}}$ to $T_{\mathrm{M}}$. A magnetic second-order phase transition occurs at $T_{\mathrm{M}}$. On the other hand, a change from a second-order to first-order magnetic phase transition at $T_{\mathrm{C}}$ takes place while $J$ becomes large from $J_{\text {crit }}$ to $J_{0}$. When we choose larger $K, T_{\text {eq }}$ becomes smaller and the transition at $T_{\mathrm{C}}$ can become of first order (see next section).

FIG. 5. The phase diagram of a SC system with magnetic interactions in the $\left(\frac{J}{K}, \frac{T}{z K}\right)$ plane. The coordinate $\left(j_{\text {crit }}, t_{\text {crit }}\right)$ denotes the end point of the reentrant phase transition. A reentrant phase transition appears for $z J_{\text {crit }}=57.6 \mathrm{~K}<z J<z J_{0}=210 \mathrm{~K}$ and magnetic order appears from $T_{\mathrm{C}}$ to $T_{\mathrm{M}}$. A magnetic second-order phase transition occurs at $T_{\mathrm{M}}$. On the other hand, magnetic transition at $T_{\mathrm{M}}$ changes from second order to first order with $J$.

Let us now discuss the case of small $J$ for which $m$ is always zero. In this case, although the magnetic interaction may cause a metastable magnetic phase at low temperatures, the system simply undergoes a transition from the diamagnetic state (LS) to the paramagnetic state (HS). This situation corresponds to the simple spincrossover phenomena. The transition can be smooth or discontinuous as the first order transition. The latter is the case of the experimental results observed in PBA, like $\mathrm{Na}_{0.44} \mathrm{Co}\left[\mathrm{Fe}(\mathrm{CN})_{6}\right]_{0.73} \cdot 2.7 \mathrm{H}_{2} \mathrm{O}[56]$.

It is worth noting that many authors have investigated the phase diagram of the original BEG model [57]. Most of works on BEG model concerned the case of 
antiferromagnetic biquadratic interaction $(K<0)[58,57,59]$ because it is expected a rich phase diagram due to competing interactions. It was found that BEG model exhibits various complicate phase transitions, such as successive phase transition, re-entrant and double re-entrant double transition against the temperature. Here, in the present study, both magnetic $(J)$ and biquadratic $(K)$ interactions are positive (ferro), and as long as these interactions are "ferromagnetic", the original BEG model does not show re-entrant phases. However, in the present study we obtain a re-entrant behavior with $J>0$ and $K>0$. On the other hand, the degeneracy of the HS state $(s= \pm 1)$ combined to the ligand field $D$ act as an effective temperature dependent "anisotropy" $\left(D-k_{\mathrm{B}} T \ln g\right)$ which is strongly reduced when temperature increases. As a consequence, in a rough approach, the original phase diagram (in the plane $t_{\mathbf{c}}-j$ at constant $D$ ) of the original BEG model (in which $g=1$ ) is changing depending on the temperature. This effect constitutes the basic mechanism of a new competition leading to obtain re-entrant phase transitions.

\section{MONTE CARLO SIMULATION}

In the previous section, we have studied the structure of the phase diagram as a function of the lattice coupling $K$ and magnetic coupling $J$ in MFT. In this section, we check the results obtained by MFT using a Monte Carlo method where the effect of fluctuation is taken into account. Here, we adopt an extended scheme of the Glauber dynamics because we study only static properties of the model. We deal with three-dimensional system $(z=6)$ with $20 \times 20 \times 20$ sites through this section.

The dynamical properties such as the relaxation process after rapid changing of temperature or photo-irradiation will be reported in the separate paper [38] where the types of dynamics will be carefully discussed.

Taking into account the degeneracy of the states, $u$ and $r$ for the low and high spin states, respectively, we adopt the following transition probability between the initial state $S$ and the final state $S^{\prime}$ : 


$$
W\left(S \rightarrow S^{\prime}\right)=\frac{P\left(S^{\prime}\right) e^{-\beta E\left(S^{\prime}\right)}}{P(0) e^{-\beta E(0)}+P(1) e^{-\beta E(1)}+P(-1) e^{-\beta E(-1)}},
$$

where $P(S)$ is the degeneracy of the electronic state, represented by the fictitious spin $S(S= \pm 1$ for the HS state and $S=0$ for the LS state). Thus, we have $P(0)=u$ and $P(1)=P(-1)=r$. Here, $E(S)$ is given by

$$
E(S)=-J S \sum_{j} S_{j}-h S-K S^{2} \sum_{j} S_{j}^{2}+\left(D-k_{\mathrm{B}} T \ln g\right) S^{2}
$$

This energy denotes the energy of the corresponding site, which can be HS or LS and it includes elastic (trough the "biquadratic" interaction) and magnetic contributions due to the interactions with the neighbors. The transition probability $W\left(S \rightarrow S^{\prime}\right)$ satisfies the detailed balance for the canonical distribution for the degenerate state.

we define the following quantity $m^{\prime}$ to study magnetic order in the MC method

$$
m^{\prime}=\frac{\left\langle\left(\sum_{i} S_{i}\right)^{2}\right\rangle}{N^{2}}
$$

where $\langle\cdots\rangle$ means MC average and $N$ is the system size $\left(20^{3}\right)$.

FIG. 6. (a) Temperature dependence of $q(\bigcirc)$ and $m^{\prime}(\triangle)$ obtained by the Monte Carlo simulation. (b) Temperature and field dependence of $q$ for the first order phase transition. Circles $(\bigcirc)$ denotes the case $h=0,(\triangle) h=9$ and $(\square) h=18$. (c) Temperature and field dependence of $m^{\prime}$ for the first order phase transition. Note that the magnetization shows non-monotonic behavior for nonzero $h$.

In Figs. 6, we show the temperature dependence of $q(\bigcirc)$ and $m^{\prime}(\triangle)$. Here the temperature is raised up from $T=5$ to $80 \mathrm{~K}$ sequentially, and is reduced to $T=5 \mathrm{~K}$ again. In Fig.6(a), smooth change of $q$ and no change of $m^{\prime}$ is depicted for $J=0$, $h=0, g=10, K=15 \mathrm{~K}$ and $D=150 \mathrm{~K}$. The transition temperature $\left(T_{\text {eq }}=35.1 \mathrm{~K}\right)$ is larger than the critical temperature $\left(T_{\mathrm{I}}=16.9 \mathrm{~K}\right)$ of the corresponding Ising model and no phase transition occurs. We find that the plotted circles for both upward and downward processes are well superimposed at each temperature. In Fig.6 (b) and (c), we plot $q$ and $m^{\prime}$ in the case of the first order phase transition, respectively, 
where $g=10, K=28 \mathrm{~K}$, and $D=150 \mathrm{~K}$, giving $T_{\text {eq }}=22.0 \mathrm{~K}\left(<T_{\mathrm{I}}=31.5 \mathrm{~K}\right)$, for $J=0, h=0(\bigcirc)$ and for $h \neq 0(\triangle, \square)$.

We performed relatively short steps (200MCS) at each temperature for Figs 6, which is enough to get equilibrium for case (a), leading well visible hysteresis loops of $q$ for case (b).

The shape of the hysteresis changes with the duration time in principle. Indeed, if we change the temperature infinitesimally slow, the system would change in a quasistatic way and the hysteresis would disappear. However, in practice, the nature of metastability is robust and the shape of the hysteresis changes little in the case with 5000 MCS at each temperature. In the Fig.6(b) we also confirm the field-dependence of the shape of the hysteresis obtained in the previous section by MFT. Triangles $(\triangle)$ denote the case $h=9$ and squares $(\square) h=18$. Shift of hysteresis curve to the low temperature side is observed and the shift is more prominent in downward process.

FIG. 7. Temperature dependence of $q(\bigcirc)$ and $m^{\prime}(\triangle)$ for $K=15 \mathrm{~K}, D=150 \mathrm{~K}, g=10$, and $J=12 \mathrm{~K}$ obtained by the Monte Carlo simulation. Inset shows the heat capacity $(\bullet)$. Note that the magnetic interaction drives the first-order SC transition.

Here, we study the effect of magnetic interaction on the spin-crossover phase transition. We consider the case where the magnetic interaction $J$ is nonzero. If we include weak magnetic interaction, the qualitative nature of the order parameter $q$ does not change, although the interaction causes a metastable branch of magnetically ordered state at low temperatures. This effect will be reported when we study the dynamical properties of the system [38]. Here, we investigate the change of equilibrium phases which were studied in the previous section in MFT. In order to change the structure of phase diagram we need a strong magnetic interaction which is comparable to $K$. In Figs.7, temperature dependence of $q$ and $m^{\prime}$ is depicted for $K=15(\mathrm{~K})$ and $D=150(\mathrm{~K})$, where $T_{\text {eq }}>T_{\mathrm{I}}$, with $J=12(\mathrm{~K})$. At each temperature, 2000 MCS are performed to obtain the data. We find that the magnetization appears 
at intermediate temperatures. Decreasing the temperature, magnetization appears continuously at the critical temperature $T_{\mathrm{M}} \simeq 43 \mathrm{~K}$ where the phase transition is of the second order as was predicted in MFT, and vanishes discontinuously in the first order phase transition at $T_{\mathrm{C}} \simeq 32 \mathrm{~K}$, where the HS fraction changes discontinuously.

FIG. 8. Phase diagrams in the coordinate $(J / K, T / z K)$ obtained by the Monte Carlo simulation. Closed squares denote $T_{\mathrm{M}}$, closed triangles $T_{\mathrm{C}}$, and closed circles the $\mathrm{SC}$ transitions. (a) for $K=15 \mathrm{~K}, D=150 \mathrm{~K}$, and $g=10$ (b) for $K=22 \mathrm{~K}, D=150 \mathrm{~K}$, and $g=10$.

Now, we study dependence of the critical temperatures $T_{M}$ and $T_{\mathrm{C}}$ as a function of $J$ for the case $K=15(\mathrm{~K}), D=150(\mathrm{~K})$ and $g=10$, where $T_{\text {eq }}>T_{\mathrm{I}}$. In Fig.8(a), the dependence is depicted, where the closed circles denote the temperature at which $q=1 / 2$. The transitions depicted by the closed circles occur smoothly. The closed triangles denote $T_{\mathrm{C}}$ and the close boxes $T_{M}$. The magnetic transition at $T_{\mathrm{C}}$ changes from second order to first order with $J$ between $J / K=0.73$ and $J / K=0.8$.

As another example, we also give the phase diagram in Fig.8(b) for the case $K=22(\mathrm{~K})$ and $D=150(\mathrm{~K})$, where $T_{\text {eq }}<T_{\mathrm{I}}$. Then the closed circles denote the phase boundary of the first order phase transition. Technically we obtain $T_{\text {eq }}$ and $T_{\mathrm{C}}$ of the first order phase transition as follows. We prepare an initial condition where half of the system is in LS state $(S=0)$ and the other half is in the magnetic HS state $(S=1)$. We perform MC simulations with different random number sequences for given $J$ and observe the relaxation of $q$ and $m^{\prime}$. When all samples go to LS (HS para, HS magnetic) phase, we regard the parameter set as belonging to the LS (HS para, HS magnetic) phase. We could determine the border within the precision of the size of the closed circles and closed triangles. These phase diagrams are very similar to those obtained by MFT in the previous section. It is interesting to study the intermediate parameter region between (a) and (b), where the line connecting the closed circles with the smooth transition (case (a)) changes to that with the first order phase transition (case (b)). 


\section{SUMMARY AND DISCUSSION}

The phase diagram of the system with the spin-crossover phase transition and the magnetic phase transition has been studied using an extended BEG model with degenerated levels by means of the mean-field theory and Monte Carlo simulations. This model can be a model for both SC and PBA materials in the framework of a common description. The present model can be also seen as an extension to 3 states of the Wajnflasz and Pick model for spin-transition [24]. This unified approach contains both "elastic" and magnetic interactions between the molecular species. These two kind of interactions obey to different symmetries, and then involve two different order parameters. Indeed, the terms responsible for the SC phenomena are the temperature-dependent ligand field $\left(D-k_{\mathrm{B}} T \ln g\right) S_{i}^{2}$ and the biquadratic $-K S_{i}^{2} S_{j}^{2}$ interaction, which modulate the energy between HS and LS states, leading to the gradual or first-order transition. On the other hand, the magnetic interaction $-J S_{i} S_{j}$, assumed here as ferromagnetic, acts only between the HS units, which breaks the symmetry, and leads to the second-order or first-order magnetic transition when it competes with the SC transition.

We found that when the magnetic interaction is weak, it does not cause a magnetic phase transition at even very low temperature at equilibrium. It only causes a metastable magnetic state at very low temperature. This is consistent with the observation in PBA. The properties of the metastable state will be reported in the study of dynamics [38]. It has also been found that the magnetic interaction causes a magnetic order in the HS phase at intermediate temperatures, i.e. $T_{\mathrm{C}}<T<T_{\mathrm{M}}$. The magnetic order disappears at a lower temperature than $T_{\mathrm{C}}$.

Even in the condition of $K$ and $D$ that $q(T)$ changes smoothly when $J=0$, i.e., $T_{\text {eq }}>T_{\mathrm{I}}$, the magnetic interaction drives the spin crossover transition which causes the first-order transition for large $J$.

As we have referred in the text, the dynamical properties of the present model are also very interesting and important in the light of recent experimental extension 
of photo magnetization. [60-63] They would be related to a description of the photomagnetic behavior of PBA, in particular, of the relaxation properties of the photoinduced magnetic state. There, the relaxation of two coupled magnetic and elastic order parameters, which have different time scales, is involved. [38]

\section{ACKNOWLEDGMENTS}

The present work was supported by Grant-in-Aid for Scientific Research from Ministry of Education, Science, Sports, Culture, and Technology of Japan, and Centre National de la Recherche Scientifique (CNRS, PICS Japon N²272) of France. One of the authors (K. Boukheddaden) is grateful for financial support for the collaboration expenses from the Grants. The numerical calculation is supported by the supercomputer center of the institute of the solid state physics of Tokyo university, which is also deeply acknowledged. 


\section{REFERENCES}

* Also at International Center For Young Scientists, National Institute for Materials Science, Tsukuba, Ibaraki 305-0044, Japan.

[1] J.H. Ammeter, Nouv. J. Chimie 4, 631 (1980)

[2] O. Kahn, Molecular Magnetism, VCH, New York, 1993.

[3] F. Varret, M. Noguès, A. Goujon, in: J. Miller, M. Drillon (Eds.), Magnetism: Molecules to Materials, vol. 2, Wiley-VCH, 2001, p. 257.

[4] M. Sorai, Chem. Soc. Jpn, 74, 2223 (2001).

[5] P. Gütlich, Y. Garcia, T. Wö̈ke, Coord. Chem. Rev. 219, 839 (2001).

[6] F. Varret, A. Bleuzen, K. Boukheddaden, A. Bousseksou, E. Codjovi, C. Enachescu, A. Goujon, J. Linares, N. Menendez, M. Verdaguer, Proc. Pure Appl. Chem. Rev. (2002) in press.

[7] M. Verdaguer, Science 272, 698 (1996).

[8] H.W. Liu, K. Matsuda, Z.Z. Gu, K. Takahashi, A.L. Cui, R. Nakajima, A. Fujishima, O. Sato, Phys. Rev. Lett. 90, 167403 (2003).

[9] E. Freysz, S. Montant, S. Létatrd, J.F. Létard, Chem. Phys. Lett. 394, 318 (2003).

[10] H. Toflund, Coord. Chem. Rev. 94, 67 (1989).

[11] E. König, Struct. Bonding 76, 51 (1991).

[12] P. Gütlich, A. Hauser, H. Spiering, Angew. Chem. Int. Ed. Engl. 33, 2024 (1994).

[13] O. Sato, T. Iyoda, A. Fujishima, K. Hashimoto, Science 272, 704 (1996).

[14] A. Bleuzen, C. Lomenech, V. Escax, F. Villain, F. Varret, C. Cartier dit Moulin, M. Verdaguer, J. Amer. Chem. Soc. 122, 6648 (2000). 
[15] A. Goujon, O. Roubeau, M. Noguès, F. Varret, A. Dolbecq, M. Verdaguer, Eur. Phys. J. B. 14, 1145 (2000).

[16] N. Kojima, W. Aoki, M. Itoi, Y. Ono, M. Sato, Y. Kobayashi and Yu. Maeda, Sokid State Commum. 120165 (2001).

[17] S. Miyashita and N. Kojima, Prog. Thore. Phys. 109729 (2003).

[18] T. Kambara, J. Chem. Phys. 70, 4199 (1979).

[19] J. Jeftic, H. Romstedt, A. Hauser, J. Phys. Chem. Solids 57, 1743 (1996).

[20] M.-L. Boillot, J. Zarembovitch, J.-P. Ities, A. Polian, E. Bourdet, J.G. Haasnoot, New. J. Chem. 25, 313 (2002).

[21] J. Jeftic, N. Menendez, A. Wack, E. Codjovi, J. Linares, A. Goujon, G. Hamel, S. Klotz, G. Syfosse, F. Varret, Meas. Sci. Technol. 10, 1059 (1999).

[22] A. Sava, C. Enachescu, A. Stancu, K. Boukheddaden, E. Codjovi, I. Maurin, F. Varret, Journal of Optoelectronics and Advanced Materials 5, 977 (2003).

[23] Y. Kobayashi, M. Itoi, N. Kojima and K. Asai, J. Phys. Soc. jpn. 713016 (2002).

[24] J. Wajnflasz, Phys. Status Solidi 40537 (1970). J. Wajnflasz and R. Pick, J. Phys. Colloq. France 32, C1 (1971).

[25] S. Doniach, J. Chem. Phys. 68, 11 (1978). M. Nielsen, L. Miao, J.H. Ipsen, O.G. Mouritsen and M.J. Zuckermann, Phys. Rev. E. 54, 6889 (1996).

[26] K. Boukheddaden, I. Shteto, B. Hôo, F. Varret, Phys. Rev. B 62, 14796 (2000); ibid. 14806 (2000).

[27] H. Spiering, E. Meissner, H. Köppen, E.W. Müller, P. Gütlich, Chem. Phys. 68, 65 (1982). N. Willenbacher, H. Spiering, J. Phys. C. 21, 1423 (1988). H. Spiering, N. Willenbacher, J. Phys. Cond. Matter. 1, 10089 (1989).

[28] J. Nasser, K. Boukheddaden, J. Linares, Eur. Phys. J. B. 39, 219 (2004). 
[29] J. Nasser, Eur. Phys. J. B. 21, 3 (2001).

[30] M. Nishino, S. Miyashita, K. Boukheddaden, J. Chem. Phys. 118, 10 (2003).

[31] A. Bousseksou, J. Nasser, J. Linares, K. Boukheddaden, and F. Varret, J. Phys. I France 2, 1381 (1992).

[32] H. Köppen, E.W. Müller, C.P. Köhler, H. Spiering, E. Meissner, P. Gütlich, Chem. Phys. lett. 91, 348 (1982).

[33] M. Nishino, K. Boukheddaden, S. Miyashita, and F. Varret, Phys. Rev. B 68, $224402(2003)$.

[34] M. Nishino, K. Yamaguchi and S. Miyashita, Phys. Rev. B 58, 9303 (1998).

[35] M. Nishino and S. Miyashita, Phys. Rev. B 63, 174404 (2001).

[36] M. Blume, Phys. Rev. 141, 517 (1966).

[37] H. W. Capel, Physica (Amsterdam) 32, 966 (1966); 33, 295 (1967); 37, 423 (1967).

[38] M. Nishino, K. Boukheddaden, S. Miyashita, F. Varret, in preparation.

[39] M. Blume, V.J. Emery, R.B. Griffiths, Phys. Rev. A. 4, 1071 (1971). K. Kasono, I. Ono Z. Phys. B.-Condensed Matter 88, 205 (1992).

[40] K. Boukheddaden, Prog. Theor. Phys. 112, 205 (2004).

[41] I. Shteto, K. Boukheddaden, F. Varret, Phys. Rev. E.60, 5139 (1999).

[42] J. Sivardière and J. Lazerowitch Phys. Rev. A. 11, 2079 (1975); J. Sivardière and J. Lazerowitch Phys. Rev. A. 11, 2090 (1975); J. Sivardière and J Lazerowitch, Phys. Rev. A. 11, 2101 (1975).

[43] T. Luty, in Relaxations of Excited States and Photo-Induced Phase Transitions, edited by K. Nasu (Springer-Verlag, Berlin, 1997), p. 142.

[44] M. H. Lemée-Cailleau, M. Le Cointe, H. Cailleau, T. Luty, F. Moussa, J. Roos, 
D. Brinkmann, B. Toudic, C. Ayache, and N. Karl, Phys. Rev. Lett. 79, 1690 (1997)

[45] S. Koshihara, Y. Tokura, T. Mitani, G. Saito, and T. Koda, Phys. Rev. B 42, 6853 (1990); S. Koshihara et al., Synth. Met. 70, 1225 (1995).

[46] F. Varret, A. Goujon, K. Boukheddaden et al., Mol. Cryst. Liq. Cryst. 379, 333 (2002).

[47] G.S. Matouzenko, G. Molnar, N. Bréfuel, et al., Chem. Mater. 15, 550 (2003).

[48] J. Linares, H. Spiering, F. Varret, Eur. J. Phys. B 10, 271 (1999).

[49] K. Boukheddaden, J. Linares, F. Varret, Eur. J. Phys. B. 15, 317 (2000).

[50] D. Mukamel, M. Blume, Phys. Rev. B. 10, 610 (1974)

[51] Y. Ogawa, T. Ishikawa, S. Koshihara, K. Boukheddaden, and F. Varret, Phys. Rev. B. 66, 073104 (2002)

[52] A. Bousseksou, N. Negre, M. Goiran, L. Salmon, J.-P. Tuchagues, M.-L. Boillot, K. Boukheddaden, F. Varret, Eur. Phys. J. B. 13, 451 (2000).

[53] A. Bousseksou, K. Boukheddaden, M. Goiran, C. Consejo, M-L. Boillot, and J-P. Tuchagues, Phys. Rev. B 65, 172412 (2002).

[54] N. Tohira, H. Okawa, S. Kida, Chem. Lett. 1979, 683 (1979).

[55] J. Zarembovitch, New. J. Chem. 16, 225 (1992).

[56] N. Shimamoto, S. Ohkoshi, O. Sato, K. Hashimoto, Inorg. Chem. 41, 678 (2002).

[57] I. Ono, J. Phys. Soc. Jpn. C8, 1541 (1988).

[58] Y.L. Wang, C. Wentworth, J. Appl. Phys. 621, 4411 (1987).

[59] T. Kaneyoshi, J. Phys. Soc. Jpn. 56, 4199 (1987).

[60] S. Ohkoshi, K. Hashimoto, J. Photochem. Photobiol., C 2, 71(2001). 
[61] T. Yokoyama, K. Okamoto, T. Ohta, S. Ohkoshi, K. Hashimoto, Phys. Rev. B. $65,64438(2002)$.

[62] H. Tokoro, S. Ohkoshi, K. Hashimoto, Appl. Phys. Lett. 82, 1245 (2003).

[63] V. Escax, A. Bleuzen, J. P. Itié, P. Munsch, F. Varret, and M. Verdaguer, J. Phys. Chem. B 107, 4763 (2003). 


\section{Energy}

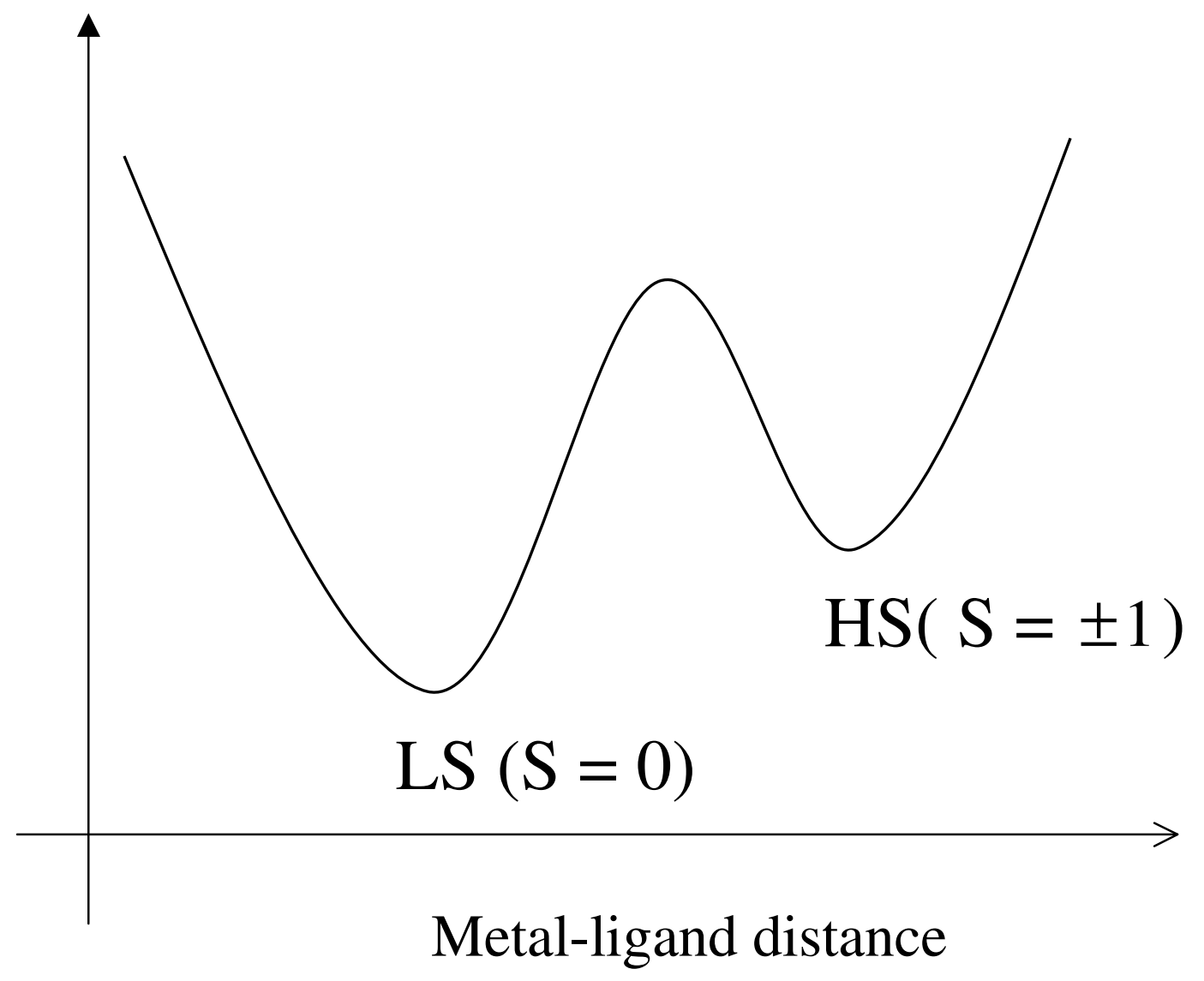

Fig.1

K. Boukheddaden, M. Nishino, S. Miyashita, and F. Varret 


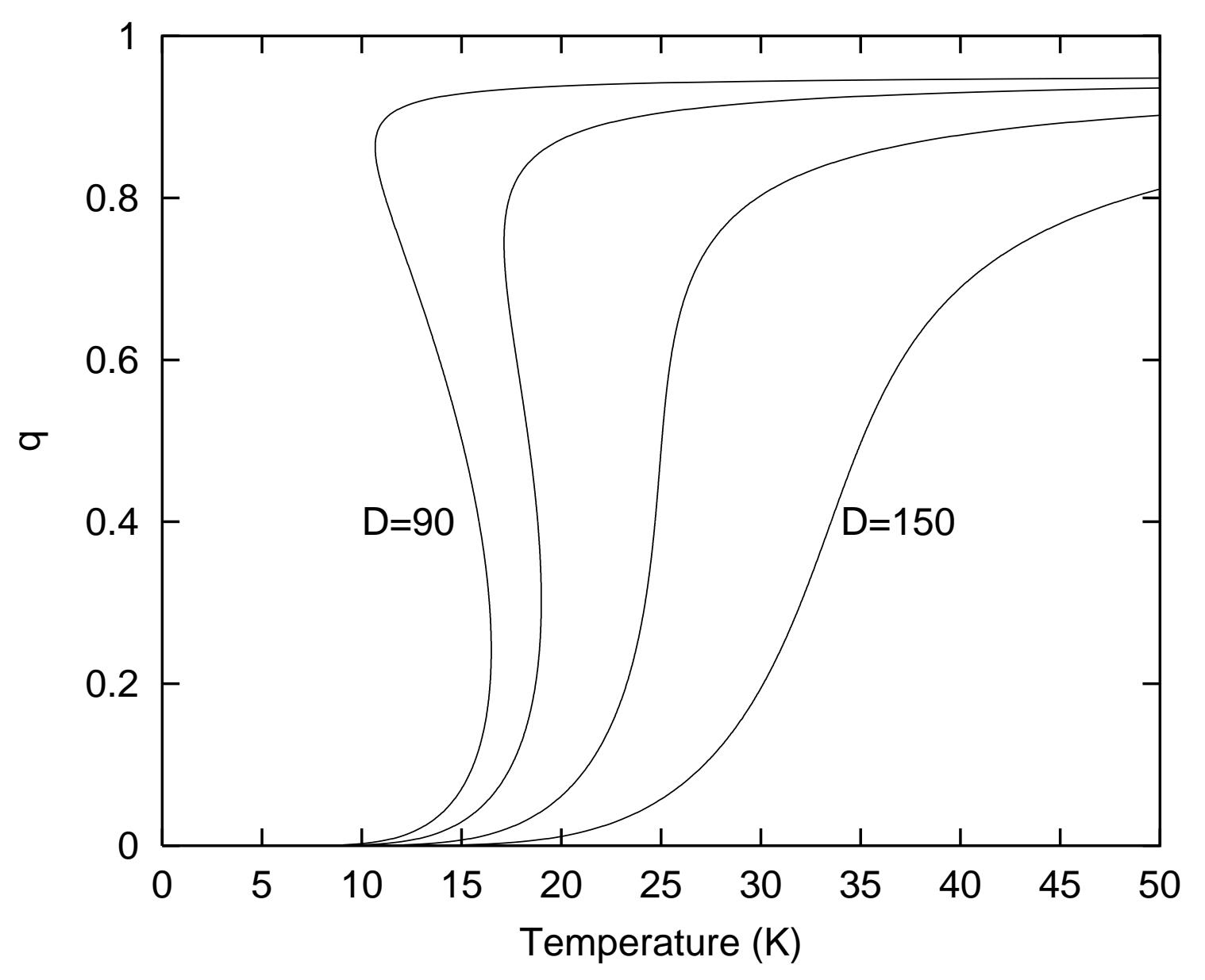




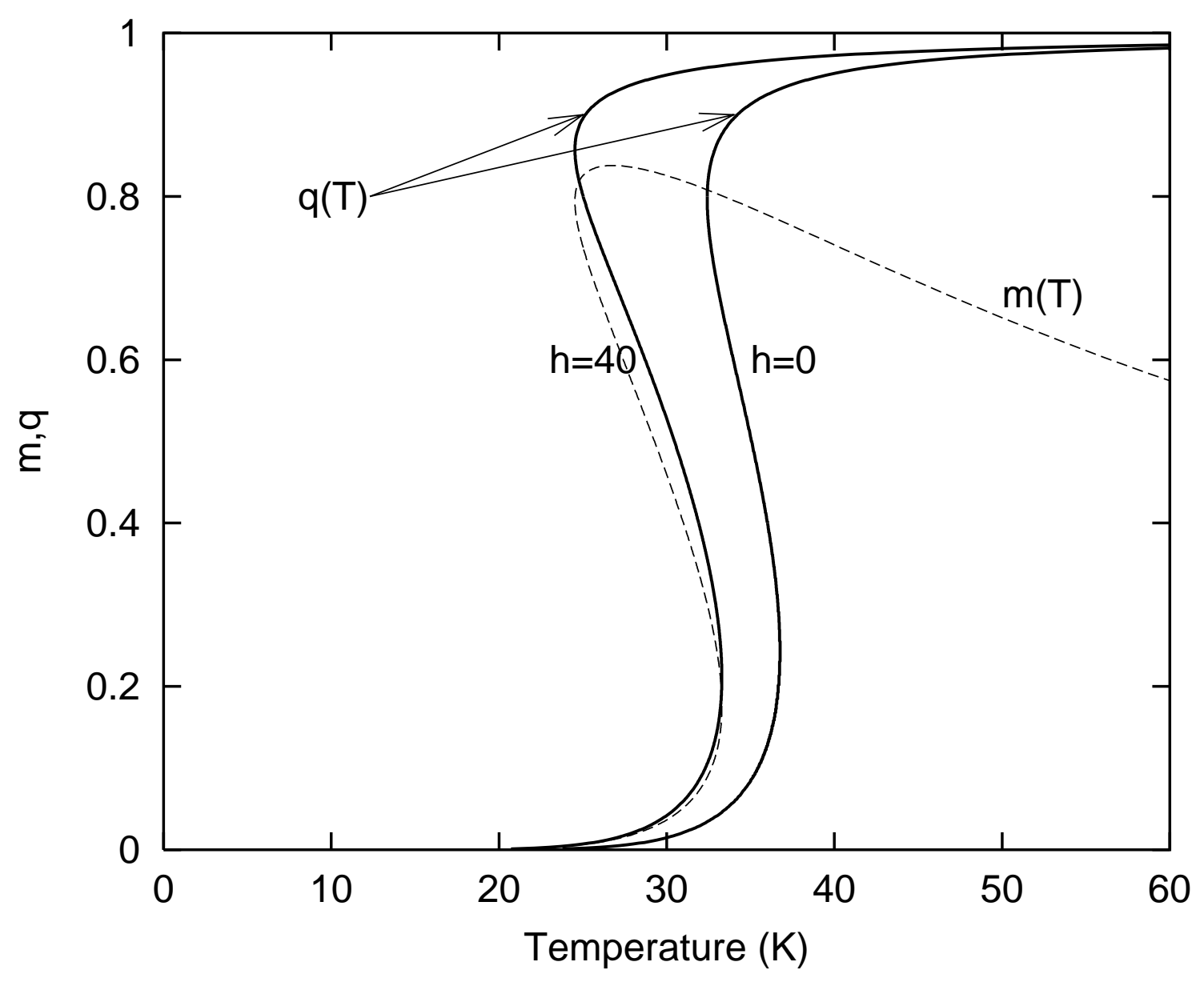




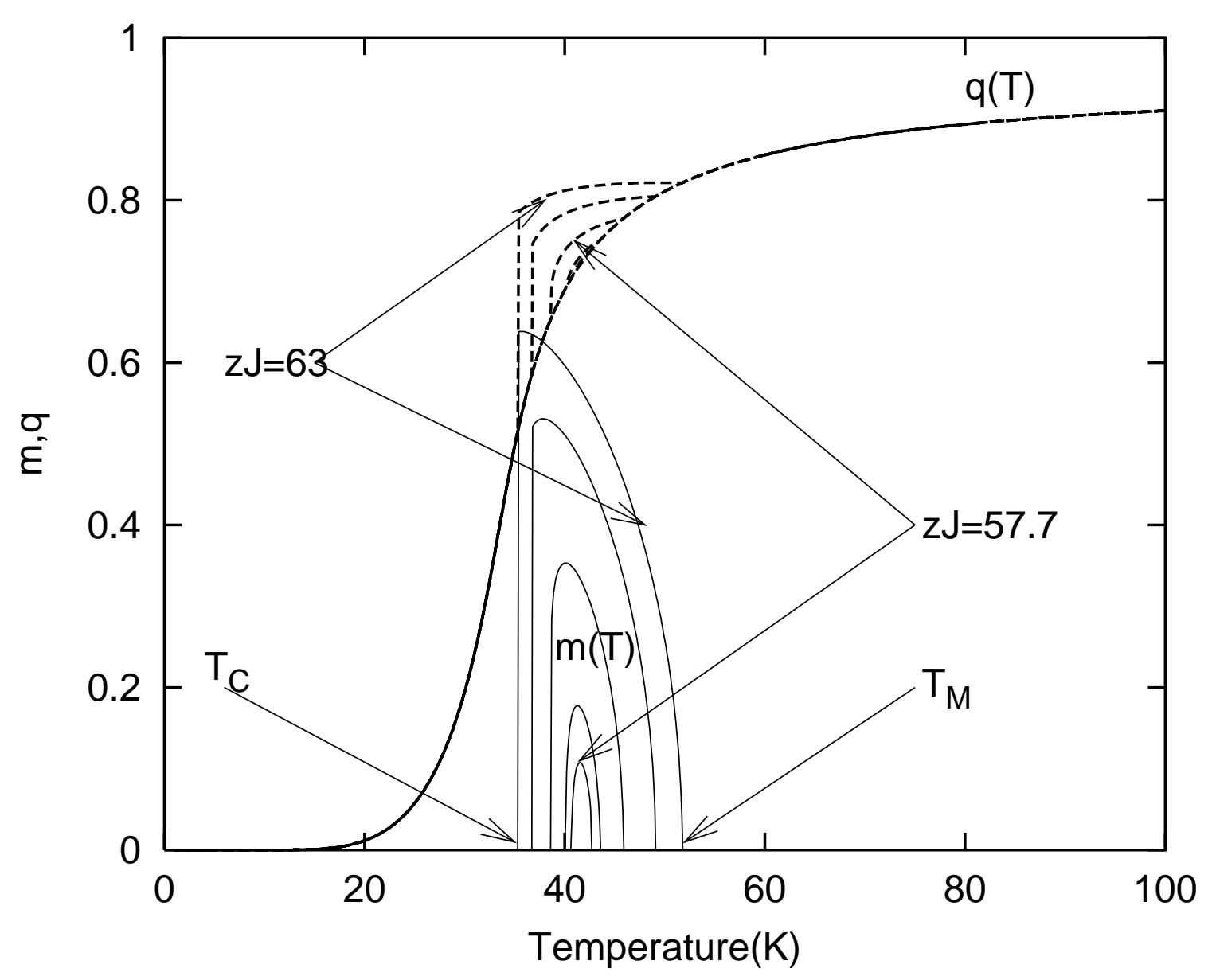




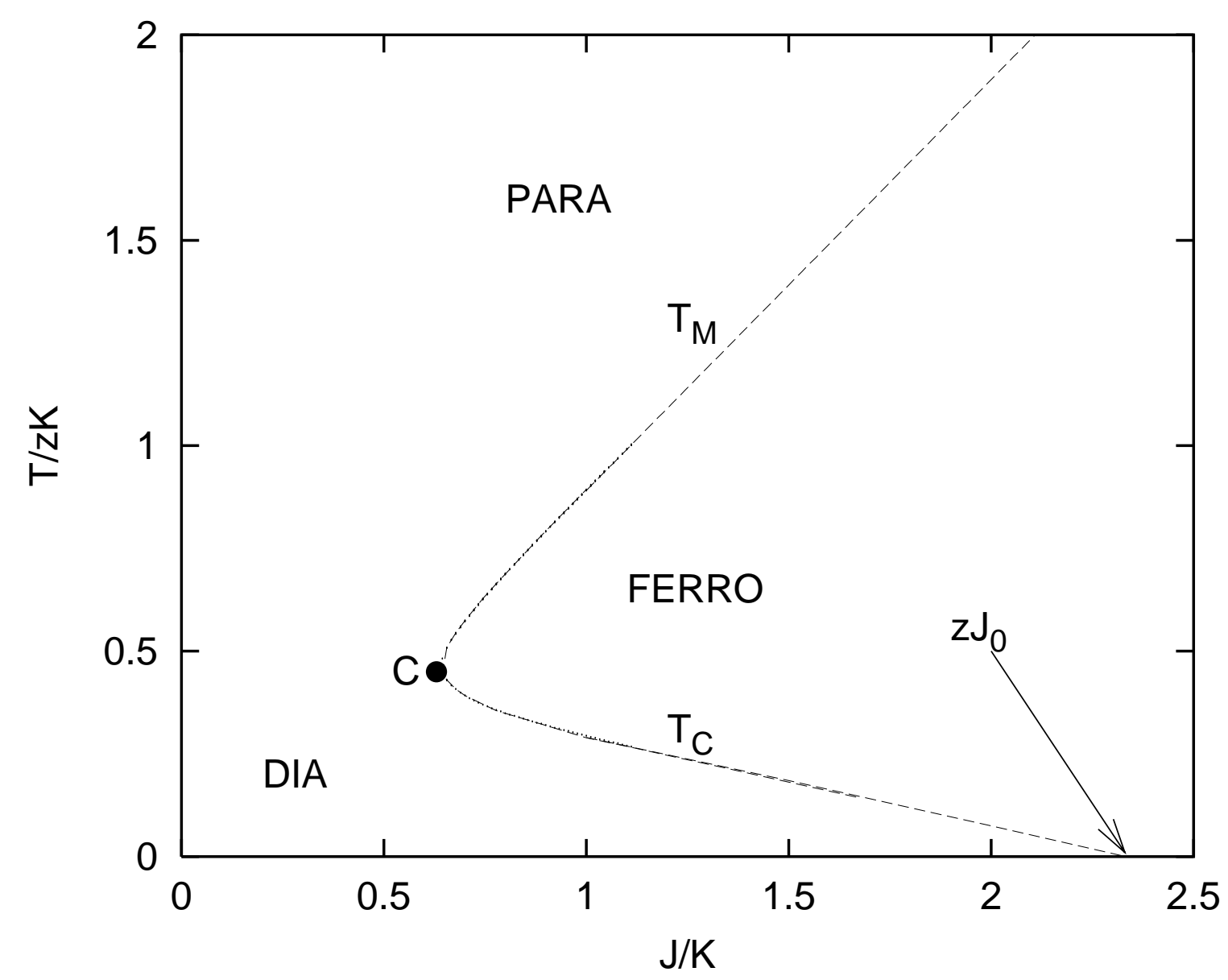




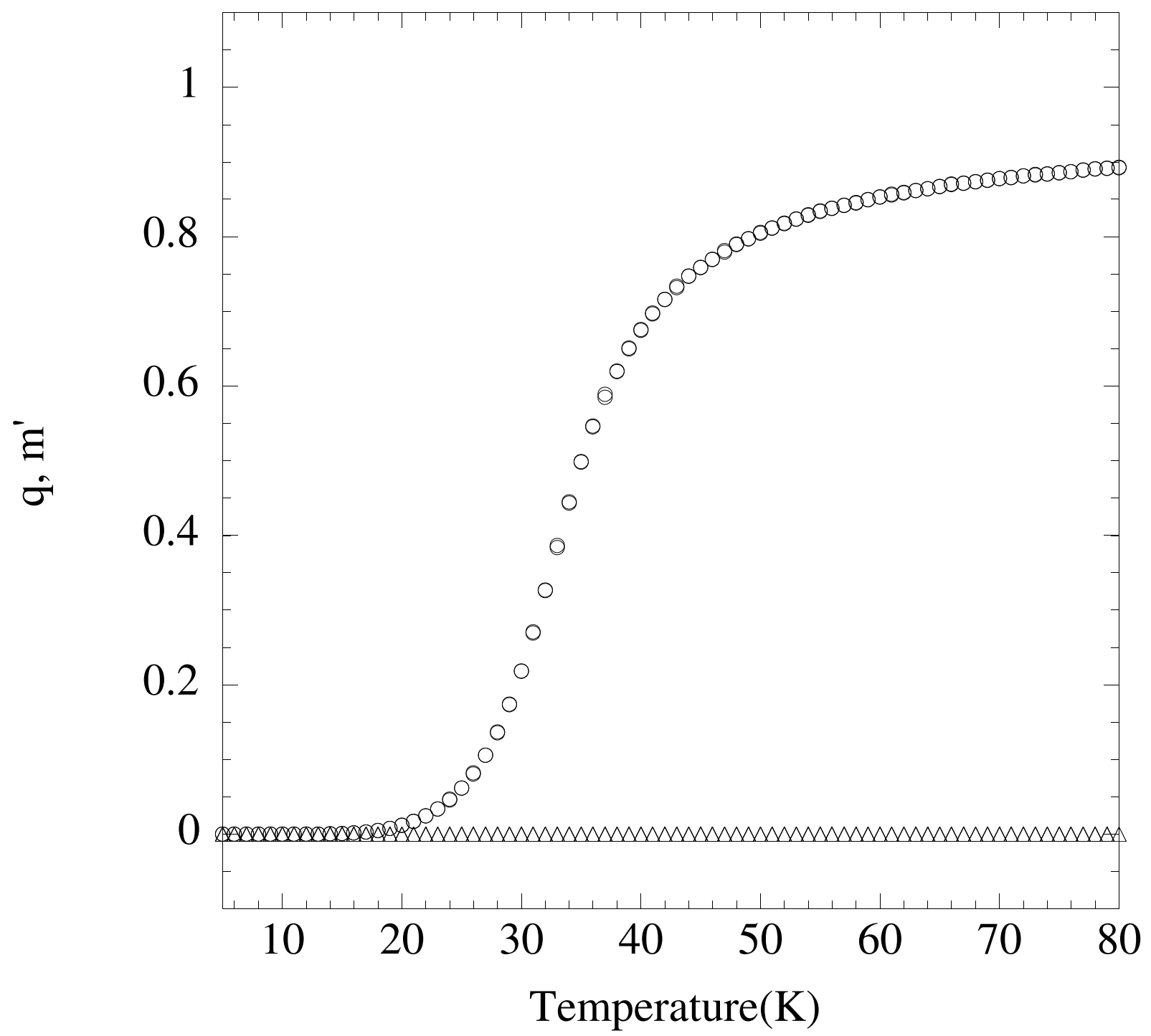

Fig.6a K. Boukheddaden, M. Nishino, S. Miyashita, and F. Varret 


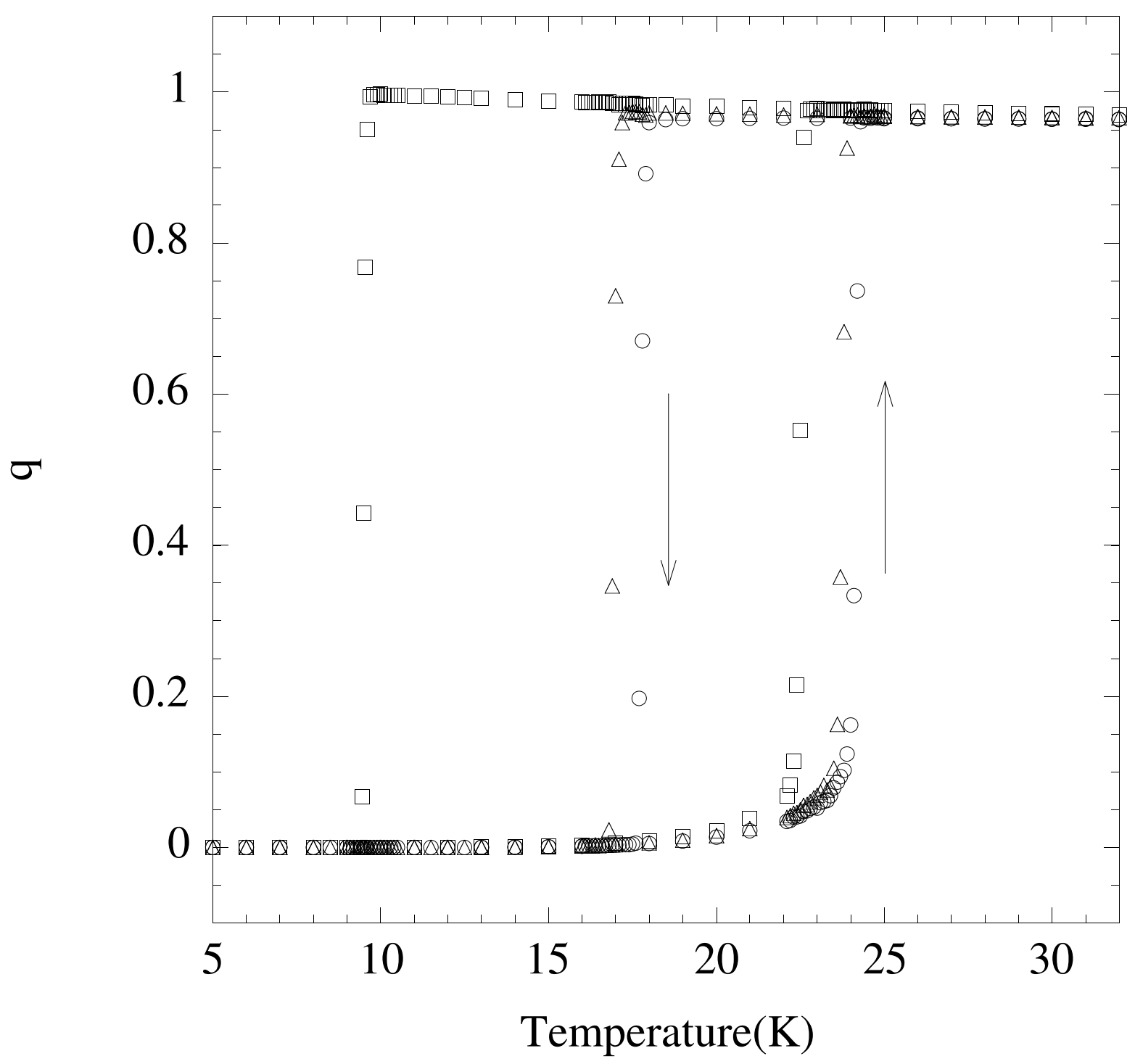

Fig.6b K. Boukheddaden, M. Nishino, S. Miyashita, and F. Varret 


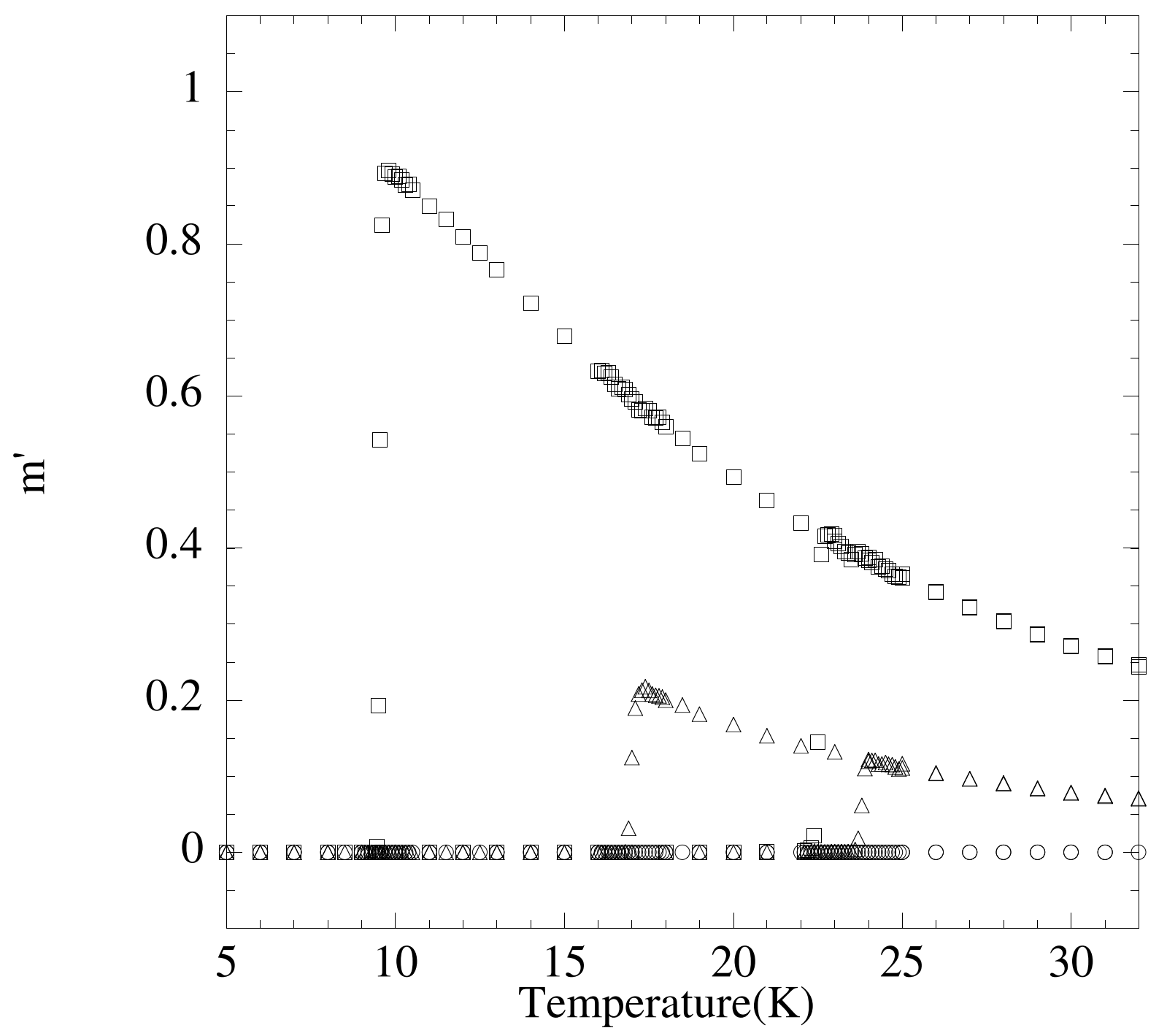

Fig.6c K. Boukheddaden, M. Nishino, S. Miyashita, and F. Varret 


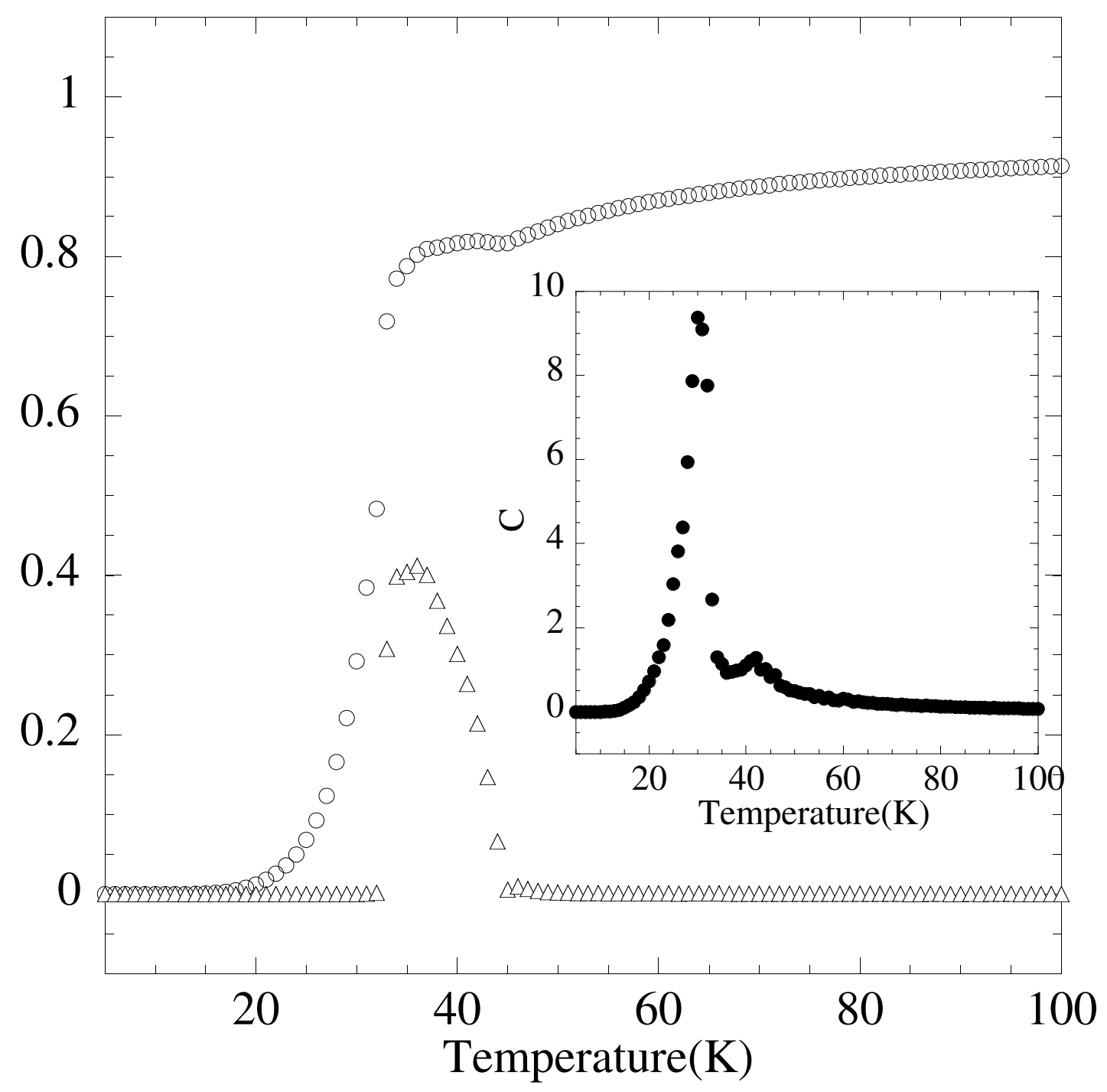

Fig.7 K. Boukheddaden, M. Nishino, S. Miyashita, and F. Varret 


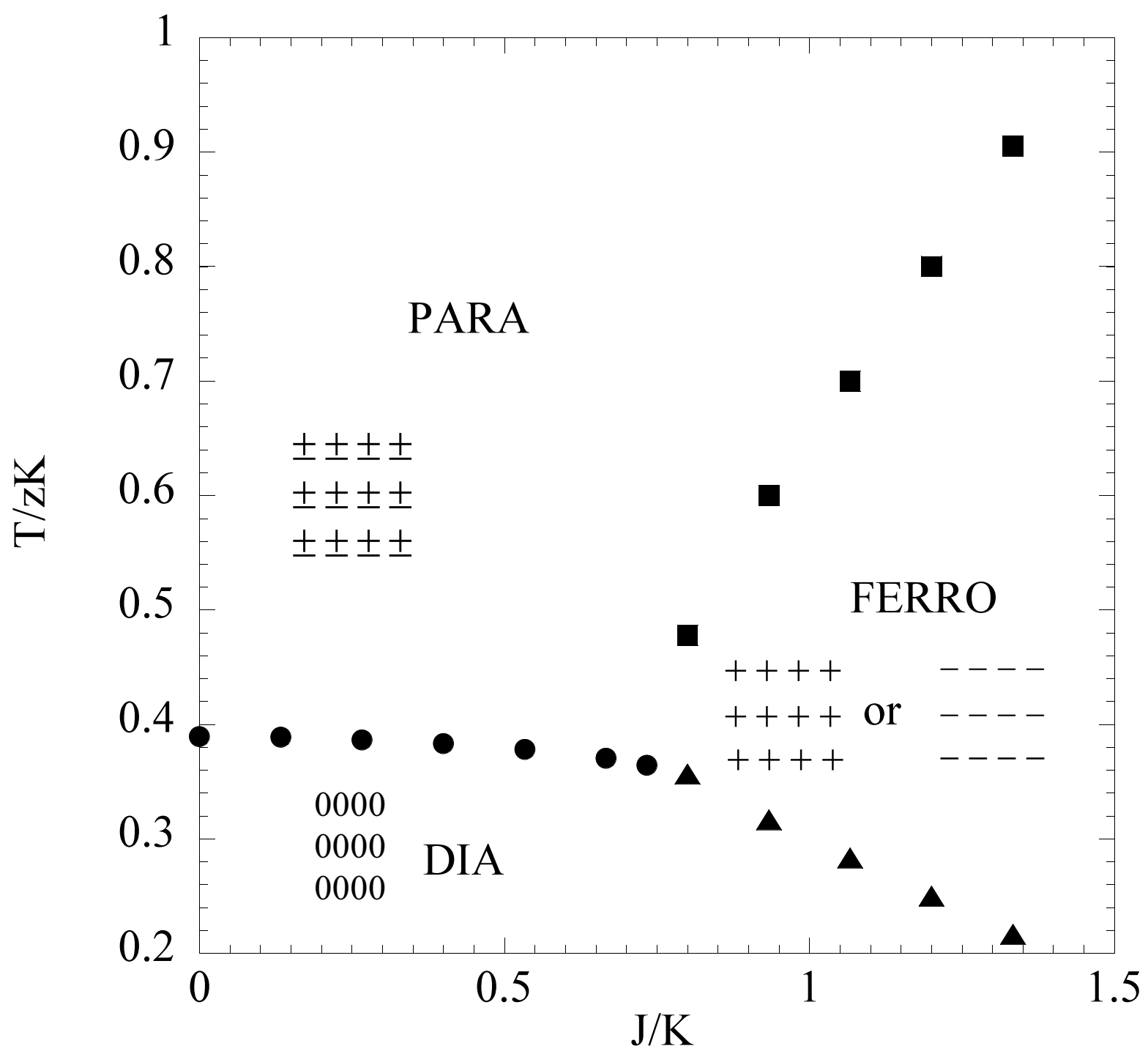

Fig.8a K. Boukheddaden, M. Nishino, S. Miyashita, and F. Varret 


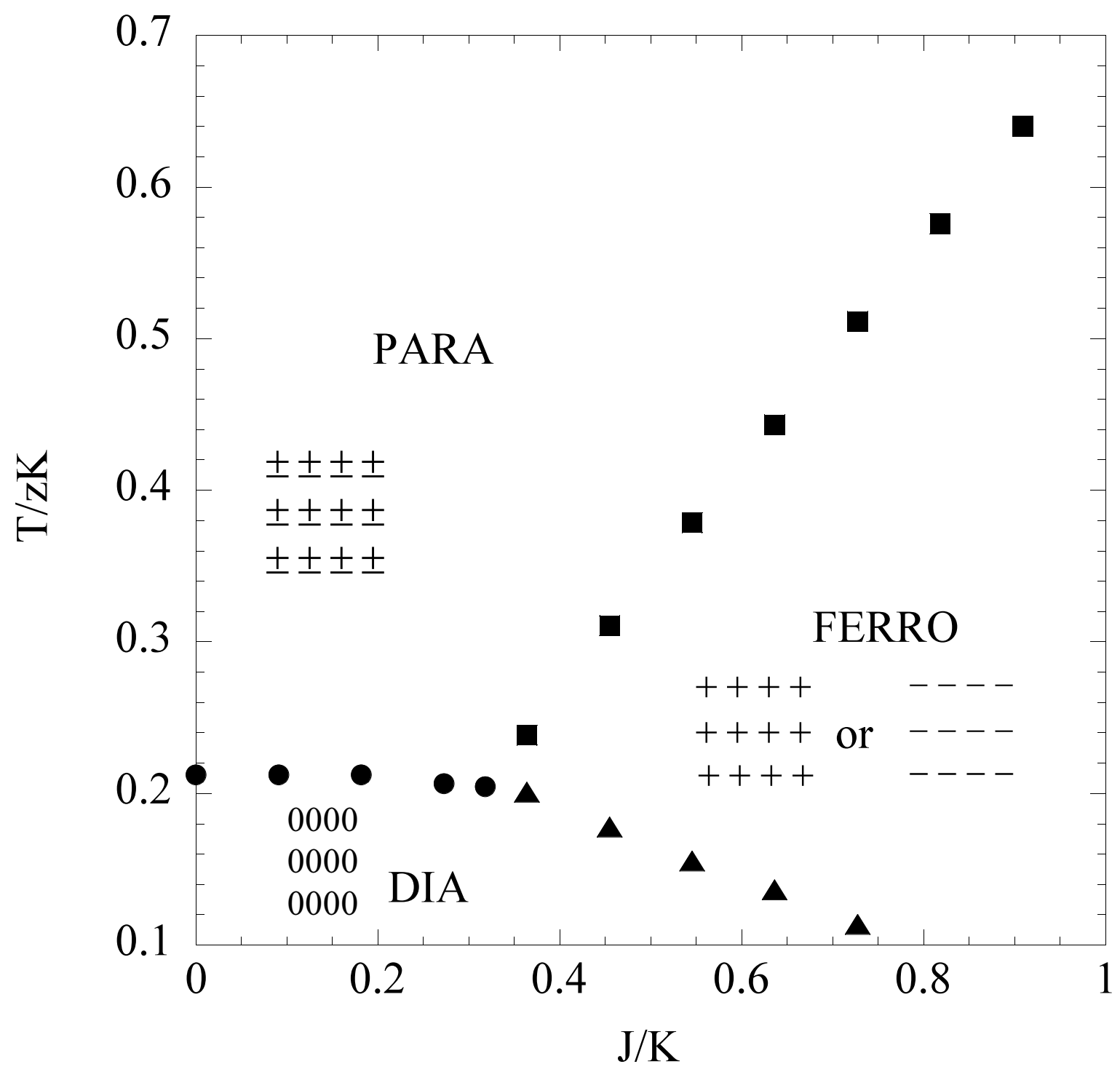

Fig.8b K. Boukheddaden, M. Nishino, S. Miyashita, and F. Varret 